\title{
Master regulatory role of p63 in epidermal development and disease
}

\author{
Eduardo Soares $^{1,2} \cdot$ Huiqing Zhou ${ }^{1,3}$
}

Received: 30 May 2017 / Revised: 16 October 2017 / Accepted: 26 October 2017 / Published online: 4 November 2017

(C) The Author(s) 2017. This article is an open access publication

\begin{abstract}
The transcription factor p63 is a master regulator of epidermal development. Mutations in $\mathrm{p} 63$ give rise to human developmental diseases that often manifest epidermal defects. In this review, we summarize major p63 isoforms identified so far and p63 mutation-associated human diseases that show epidermal defects. We discuss key roles of p63 in epidermal keratinocyte proliferation and differentiation, emphasizing its master regulatory control of the gene expression pattern and epigenetic landscape that define epidermal fate. We subsequently review the essential function of p63 during epidermal commitment and transdifferentiation towards epithelial lineages, highlighting the notion that p63 is the guardian of the epithelial lineage. Finally, we discuss current therapeutic development strategies for p63 mutation-associated diseases. Our review proposes future directions for dissecting p63-controlled mechanisms in normal and diseased epidermal development and for developing therapeutic options.
\end{abstract}

Keywords Epidermis - Gene regulation - Ectodermal dysplasia $\cdot$ Epidermal cell identity $\cdot$ Epigenetics

Huiqing Zhou

jo.zhou@ radboudumc.nl; j.zhou@ @science.ru.nl

Eduardo Soares

e.soares@science.ru.nl

1 Department of Molecular Developmental Biology, Faculty of Science, Radboud Institute for Molecular Life Sciences, Radboud University, 274, Postbus 9101, 6500HB Nijmegen, The Netherlands

2 CAPES Foundation, Ministry of Education of Brazil, Brasília, Brazil

3 Department of Human Genetics, Radboud University Medical Center, 855, Postbus 9101, 6500HB Nijmegen, The Netherlands

\section{Introduction}

The transcription factor p63, encoded by the TP63 gene, belongs to the $\mathrm{p} 53$ gene family. Distinct from the leading member of the gene family, p53, that plays an important role in tumor suppression, the role of p63 in cancer is not fully understood. However, p63 has been shown to be a key regulator of epidermal development. This has been demonstrated in various animal models and by p63 mutation-associated human diseases. For example, complete deletion of p63 in mice results in the absence of the epidermis and epidermal related appendages, as well as defects in other epithelialrelated tissues [1-3]. In humans, heterozygous mutations in TP63 cause several developmental disorders, and many of these diseases manifest skin abnormalities [4-7]. Classical studies showed that p63 is an important player in embryonic epidermal development and in epidermal keratinocyte proliferation and differentiation, where it directly regulates numerous target genes involved in cell proliferation, differentiation and adhesion [8-10]. Several recent studies demonstrated that p63 also plays a role in the modulation of the epigenetic and chromatin landscape in epidermal keratinocytes by directly regulating chromatin factors and by engaging and opening chromatin regions [11-16]. Among these studies, those using unbiased genome-wide approaches convincingly established that p63 is a key regulator controlling the enhancer landscape. These recent findings suggest a more sophisticated model of the master regulatory role of p63 and reveal additional layers of complexity in p63-orchestrated gene regulation of epidermal development and related diseases.

Different p63 isoforms have been shown to play roles in various cells and tissues, such as the epidermis, oocytes, muscles and cochlea. As such, this review discusses p63 isoforms identified so far, with a focus on isoforms that are 
expressed in epidermal cells. Subsequently, we dedicate most of this review to the most studied topic in p63 biology: the role of p63 in epidermal development and related diseases. Specifically, we provide an overview of p63 mutation-associated developmental diseases with epidermal phenotypes. We discuss in-depth key molecular and cellular mechanisms by which $\mathrm{p} 63$ controls gene regulation to define epidermal identity, and speculate that the affected cell fate contributes to various disease states. Finally, we review current efforts and future perspectives in developing therapeutic strategies for treating these diseases.

\section{p63 isoforms and their expression}

The transcription factor p63 was initially described as keratinocyte transcription factor (KET) in 1997, as it is homologous to p53 in rat epithelial tissues [17]. One year later, it was denoted as one of the p 53 gene family members in a comprehensive cloning study [2]. In this study, six isoforms resulting from two alternative promoters (TA and $\Delta \mathrm{N}$ ) and three different splicing routes $(\alpha, \beta, \gamma)$ were identified from mouse E15 embryos and a human neuroepithelioma cell line [2], indicating that these isoforms were expressed in those cells and tissues. The TA isoform contains three TA-specific exons, exon 1, 2 and 3, and encodes a transactivation domain (TA1) that is homologous to the transactivation domain of the p53 protein (Fig. 1). Another promoter is used to produce a shorter isoform denoted $\Delta \mathrm{N}$, and its starting exon, named as exon $3^{\prime}$, is specific to the $\Delta \mathrm{N}$ isoform. Initially, the $\Delta \mathrm{N}$ isoform was considered transcriptionally inactive, functioning as a dominant negative variant towards the TA isoform, because it lacks the typical transactivation domain TA1. However, it has since been recognized that the $\mathrm{N}$-terminal region of the $\Delta \mathrm{N}$ isoform also possesses transactivation activity, and therefore it is termed as $\mathrm{TA}^{\Delta \mathrm{N}}$ (Fig. 1). At the $\mathrm{C}$-terminus, the longest isoform is the $\alpha$ isoform that contains all 3' exons 11-14 (Fig. 1). At the protein level, the $\alpha$ isoform contains a sterile alpha-motif (SAM) domain that is thought to be involved in protein-protein interactions [2] and a Transactivation Inhibitory Domain (TID) that inhibits the activity of the TA1 domain [18]. Two other isoforms, $\beta$ that lacks the exon 13 and $\gamma$ that does not have exons 11-14 but has a $\gamma$-specific exon $10^{\prime}$, do not contain the SAM and TID domains.

In recent years, several novel isoforms have been reported. At the $\mathrm{N}$-terminal end of the $\mathrm{p} 63$ protein, an alternative translational start site located in the 4th exon gives rise to a $\Delta \Delta \mathrm{N}$ isoform that lacks the first 26 amino acids of the $\Delta \mathrm{N}$ isoform in epidermal keratinocytes (Fig. 1). This isoform was identified by Rinne et al. [19]. In their study on AEC/RHS syndrome, stop mutations were found within the first 26 amino acids of p63 in several AEC/RHS patients. To the surprise of the authors, the TP63 transcript was detected, rather than being degraded completely via the nonsensemediated decay mechanism that is common for stop mutations. This incidental observation led to the identification of an alternative translational start site downstream of all stop mutations. This alternative translational start site gives rise to the $\Delta \Delta \mathrm{N}$ protein variant in AEC/RHS patient keratinocytes, and it is also present in keratinocytes of healthy controls, although at a lower level than the $\Delta \mathrm{N}$ isoform. At the $\mathrm{C}$-terminal end, two other isoforms, $\delta$ and $\varepsilon$, were identified by bioinformatic analyses using the Alternative Splicing
Fig. 1 Gene and protein structures of p63 and mutations involved in developmental syndromes with epidermal phenotypes (ectodermal dysplasia). Two promoters resulting in $\mathrm{N}$-terminal TA and $\Delta \mathrm{N}$ isoforms are indicated with arrows. Exons and protein domains are numbered and color-coded as indicated. p63 mutation-associated ectodermal dysplasia syndromes are marked in round-edge rectangles and the locations of their associated hotspot mutations are indicated by black lines. The main phenotypes related to the syndromes are shown near the rectangles

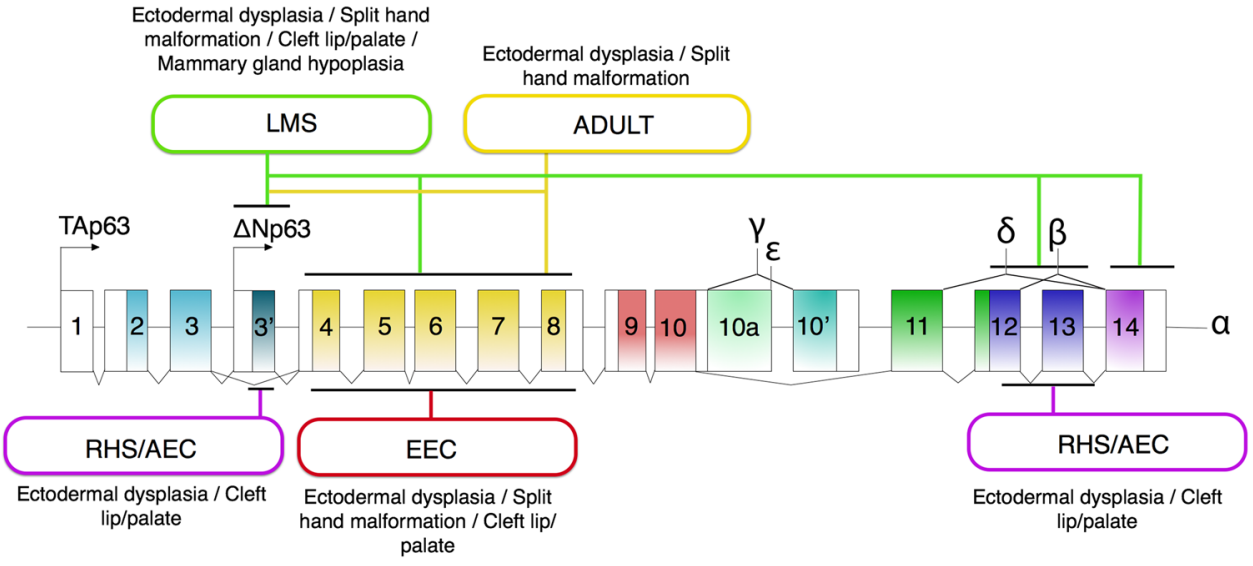

TA1 transactivation domain

$\mathrm{TA}^{\mathrm{\Delta N}}$ transactivation domain

DNA binding domain
Oligomerization domain

Transactivation inhibitory domain

TA2 transactivation domain

Strile alpha motif 
Prediction Data Base, ASPicDB (http://www.caspur.it/ ASPicDB/index.php) and validated by reverse transcription quantitative PCR (RT-qPCR) in several cell lines including keratinocytes, HEK293 cells and in some tissues, such as the muscle and the brain [20]. The $\delta$ isoform is an alternatively spliced variant that lacks exon 13 . The $\varepsilon$ isoform is generated by a premature stop codon that is located in exon 10. These isoforms were later validated by an RNAseq analysis in mouse keratinocytes [21]. In the same mouse keratinocyte RNA-seq study, the authors additionally identified a slightly shorter isoform that lacks four amino acids encoded by nucleotides located in exon 8 . Furthermore, in a p63 isoform analysis using RNA-seq data from squamous cell carcinoma (SCC) cell lines and from publically available data from cells of all three germ layer origins, Sethi et al. confirmed all previously reported p63 isoforms in different cell types [22]. Isoforms identified so far all contain the DNA-binding domain (DBD) and the oligomerization domain (OD) (Fig. 1).

Expression patterns and functions of TA and $\Delta \mathrm{N}$ isoforms during epidermal commitment and subsequent stratification have been under debate, although it is generally agreed that the $\Delta \mathrm{N}$ isoform is highly expressed in basal epithelial cells. One report showed that the TA isoform was expressed first and subsequently required to initiate epidermal stratification, but it was counter-balanced by the $\Delta \mathrm{N}$ isoform during stratification [23]. In contrast, another study showed the $\Delta \mathrm{N}$ isoform was required for the proliferation of epidermal cells in the basal layer, while the TA isoform was required for activating genes during stratification [24]. These studies proposed an interesting switch of the two isoforms during epidermal development. However, these observations have not been confirmed by other studies. The majority of current literature agrees that the $\Delta \mathrm{N}$ isoform is the major functional isoform in epithelial cells and tissues, such as oral and dental tissues, corneal tissues and lung epithelial cells [1, 3, 25-28]. This scenario is supported by phenotypes presented in $\Delta$ Np63-specific knockout mouse models that, similar to mice that lack all p63 isoforms, showed defects of the skin, oral epithelium, mammary glands, limb and craniofacial regions [27].

As recently developed genomic approaches allow detecting active promoters and expressed exons, these techniques greatly facilitate the identification of isoform expression at the transcript level. Several independent analyses using RNA-seq, ChIP-seq of RNA polymerase II, DNA hypersensitivity sites (DHS)-seq and cap analysis gene expression (CAGE) $[14,22,29]$ showed that the $\Delta \mathrm{N}$ promoter is the only active promoter and the $3^{\prime}$ exon is the first expressed exon detected in epidermal cells throughout epidermal stratification. Furthermore, in line with the current literature mentioned above, Sethi et al. reported that the $\Delta \mathrm{N}$ isoform is the only abundantly expressed isoform in many epithelial cells, such as those from oral tissues and the mammary gland among 40 human cell types [22]. In contrast to the abundant expression of the $\Delta \mathrm{N}$ isoform in epithelial cells, the TA isoform is generally expressed at a low level in a range of non-epithelial cells. It is expressed at a low to moderate level in Burkitt Lymphomas (BL) cell lines and the lymphoblastoid cell line GM12878 [22]. Although the findings from novel genomic analyses $[14,22,29]$ do not exclude the possibility that the TA isoform is expressed at a very low level below the detection threshold, they do cast doubts on the importance of the TA isoform is in epithelial cells, given that its expression is at least several magnitude lower than the $\Delta \mathrm{N}$ isoform. Interestingly, the TA isoform has been shown to play roles in various organs and tissues other than the epidermis. TAp63 is expressed in oocytes and plays an important role in controlling apoptosis in response to DNA damage [28]. In the cochlea, the TA isoform is also expressed and regulates the Notch signaling pathway, which is required for proper cochlea development [30]. Additionally, TAp63 has been found to be expressed in late-stage myogenesis [31] and in cardiomyocyte development [32]. In general, the TA isoform seems to play a role in stress- or condition-induced response and in senescence, aging and metabolism [20, 27-30]. Many of these reported functions of the TA isoform are consistent with phenotypes observed in TA-specific mouse models [33-35], and accordingly, these phenotypes of TA-specific mouse models are distinct from the strong epidermal, orofacial and limb phenotypes of the p63 $\Delta \mathrm{N}$-specific or complete knockout mouse models [1, $3,27]$.

As for $\mathrm{p} 63 \mathrm{C}$-terminal isoforms, the $\alpha$ isoform is the predominant one in most p63-expressing cells [22], although $\beta$, $\delta$ and $\varepsilon$ isoforms are also expressed at a low level [20,22]. The $\gamma$ isoform is reported to be expressed in muscle cells $[20,31]$, immortalized cancer cells, such as MCF7 [20] and squamous cell carcinoma cell lines [22]. As the $\alpha$ isoform is by far the most abundant isoform in cells detected so far, it has been proposed that the bulk of the $\mathrm{p} 63$ functional activity is driven by the $\alpha$ isoform [22]. Further biochemical and functional in vivo analyses of other isoforms will be important for dissecting their function.

\section{p63 mutation-associated diseases with ectodermal dysplasia}

Although p63 isoforms are expressed in a range of tissues of different germ layer origins, germline mutations of TP63 have until now only been associated with ectodermal-related disorders manifested with three hallmark defects: ectodermal dysplasia, limb malformation and orofacial clefting [5]. These diseases include Ectrodactyly, Ectodermal Dysplasia, and Cleft lip/palate syndrome (EEC, OMIM 604292), 
Ankyloblepharon-Ectodermal defects-Cleft lip/palate (AEC, OMIM 106260), Limb Mammary Syndrome (LMS, OMIM 603543), Acro-Dermato-Ungual-Lacrimal-Tooth syndrome (ADULT, OMIM 103285), Rapp-Hodgkin Syndrome (RHS, OMIM 129400). There is good genotype-phenotype correlation in p63 mutation-associated syndromes. For examples, p63 mutations associated with EEC syndrome are exclusively found in the DNA-binding domain of the protein, and those associated with AEC or RHS syndrome are located either at the $\mathrm{N}$-terminal $\mathrm{TA}^{\Delta \mathrm{N}}$ domain or C-terminal TID or SAM domain of the $\Delta \mathrm{Np} 63 \alpha$ isoform [5] (Fig. 1).

Among these disorders, EEC syndrome is the prototype of p63 mutation-associated disorders, as it exhibits defects of all three hallmarks. About $30 \%$ of EEC patients have skin defects, and the skin is often thin and dry and sometimes resembles dermatitis. Other affected tissues with ectodermal origin include hair, teeth, nails, and lacrimal ducts (Table 1). These phenotypes are reminiscent of those of p63 $\Delta \mathrm{N}$-specific or complete knockout mouse models [5], although in less severe forms. More than $90 \%$ of EEC syndrome patients carry heterozygous mutations in the p63 DNA-binding domain with a few hot spots of amino acids residues R204, R227, R279, R280, and R304. Transient transfection assays and in vitro DNA-binding assays have shown that these mutations disrupt p63 binding to DNA and act in a dominant negative fashion [36-38]. Two other syndromes with p63 mutations in the DNA-binding domain are LMS and ADULT syndromes. Both syndromes have mammary gland hypoplasia (Table 1). However, LMS patients rarely have defects in their skin and hair phenotypes, whereas ADULT patients have skin, nail and teeth defects. LMS mutations are also found in $\mathrm{N}$-terminal $\mathrm{TA}^{\Delta \mathrm{N}}$ and C-terminal SAM domains of p63. For ADULT syndrome, the amino acid R298 in the DNA-binding domain is the most significant hot spot of mutations [39]. Rather than the dominant negative model that is proposed for EEC mutations, R298 mutations implicated in ADULT syndrome are proposed to be gain-of-function [36]. Another reported ADULT syndrome mutation is $\mathrm{N} 6 \mathrm{H}$, located in the $\Delta \mathrm{N}$-specific domain of p63 (Fig. 1) [5].
Among all p63 mutation-associated syndromes, the most severe skin phenotypes are observed in AEC/RHS syndromes (Table 1). About $70-75 \%$ patients have severe skin erosions that sometimes resemble second-degree burns [40]. Defects of nails and teeth are also common phenotypes in AEC/RHS patients. In general, the ectodermal dysplasia phenotypes are milder in RHS, compared to AEC. Mutations involved in AEC/RHS syndromes, either in the $\mathrm{N}$ - or C-terminal region of the $\Delta \mathrm{Np} 63 \alpha$, have been shown to disrupt the transactivation activity of the $\Delta \mathrm{Np} 63 \alpha$ isoform in a dominant fashion.

It is generally believed that dominant negative or gain-offunction is the disease mechanism of TP63 mutations. This notion is supported by several lines of evidence. First, TP63 mutations associated with human syndromes are heterozygous, and mostly missense mutations [5]. In a disease case where the complete TP63 gene was lost in a larger genomic deletion region, the patient did not present any ectoderm dysplasia phenotype [41]. Second, studies using in vitro biochemical methods, such as transient transfection, DNA-binding assays and molecular structure modeling demonstrated that mutant $\mathrm{p} 63$ has dominant negative or gain-of-function effect towards the wild-type p63 [36, 38, 39]. Third, heterozygous p63 knockout mouse models do not exhibit any epidermal phenotype. To model the dominant negative or gain-of-function effect of TP63 mutations in human disease, two knock-in mouse models carrying Trp63 (encoding p63 in mice) mutations recapitulating EEC (R279H in human) [42] and AEC (L514F in human) [43] were reported. In the EEC knock-in model, one mouse allele carried a floxed neomycin cassette, and is herein referred to as Trp6 ${ }^{\mathrm{R} 279 \mathrm{HN}}$. Heterozygous $\operatorname{Trp} 63^{\mathrm{R} 279 \mathrm{HN}}$ mice exhibited phenotypes that are similar to those observed in EEC syndrome patients. This includes cleft palate, anomalies of the distal limbs, defective tooth morphogenesis, and dystrophic nails. Although no major skin abnormalities were detected in Trp63 $279 \mathrm{HN}$ mice, primary keratinocytes from these mice exhibit reduced proliferation and increased senescence. Furthermore, an increased level of p63 mRNA and protein was detected in Trp63 ${ }^{\mathrm{R} 279 \mathrm{HN}}$ mice, which is consistent with observations reported in the epidermis of p63 EEC patients [38]. The

Table 1 Phenotypes of skin and ectodermal-derived appendages in p63 mutation-associated syndromes

\begin{tabular}{|c|c|c|}
\hline & Skin & Ectodermal-derived appendages \\
\hline EEC syndrome & Mild phenotype-dry and thin skin & Hair, nails, teeth and glands defects- highly variable severity \\
\hline Limb mammary syndrome (LMS) & Rarely detected & $\begin{array}{l}\text { Nails and lacrimal ducts defects, hypohydrosis, nipple hypoplasia and } \\
\text { orofacial clefting }\end{array}$ \\
\hline ADULT syndrome & Dry skin-milder than EEC & Teeth, nail and lacrimal duct defects \\
\hline AEC syndrome & Severe skin erosions & $\begin{array}{l}\text { Eyelid fusion, teeth and hair defects and/or alopecia, lacrimal duct } \\
\text { obstruction and orofacial clefting }\end{array}$ \\
\hline Rapp-Hodgkin syndrome (RHS) & Severe skin erosions & $\begin{array}{l}\text { Teeth and hair defects and/or alopecia, lacrimal duct obstruction and } \\
\text { orofacial clefting }\end{array}$ \\
\hline
\end{tabular}


heterozygous AEC L514F mouse model presents hypoplastic and fragile skin, ectodermal dysplasia and cleft palate, resembling defects observed in AEC syndrome patients [5]. These phenotypes probably result from impaired FGF signaling, as two p63 direct target genes Fgfr2 and Fgfr3 showed reduced expression [43].

\section{p63-dependent gene regulation in epidermal keratinocytes}

Due to the striking epidermal phenotype of p63 knockout mouse models and of p63 mutation-associated human diseases, the molecular and cellular role of p63 in epidermal keratinocytes has been extensively studied. It has been shown that p63 is important in both keratinocyte proliferation and differentiation [10, 44]. Higher p63 expression level is found in more proliferative cells, such as holoclones that represent epidermal stem cells [9], indicating that p63 is important for keratinocyte proliferation. Many studies have used siRNA knock-down approaches to investigate the role of p63 in proliferation and differentiation. Knock-down of p63 in primary keratinocytes gives rise to hypoplasia, hypoproliferation, cell cycle arrest [10] and abnormalities in cell adhesion [8]. Furthermore, downregulation of p63 prevents cells from differentiating and stratifying in both $2 \mathrm{D}$ and $3 \mathrm{D}$ keratinocyte differentiation models [10]. Although proliferation and differentiation are related, p63 seems to have independent roles in both processes. This was supported by a study where p63 knock-down caused both hypoplasia and differentiation defects, and concomitant p53 knock-down can rescue only the hypoplasia deficiency but not differentiation defects [10]. At the molecular level, knock-down of p63 induces genes that control cell cycle arrest, such as p21 (CDKN1A) [10, 45, 46], and genes that negatively regulate cell proliferation, such as JunB [47]. At the same time, knock-down of p63 downregulates genes that can positively regulate cell proliferation, such as Fos and c-Jun [47], and genes that are important for epidermal differentiation, such as Perp and K14 [48, 49]. Consistently, many genes that are involved in cell proliferation, cell cycle control, and keratinocyte differentiation have been shown to be direct target genes of p63 (see review [50]). In addition to controlling cell cycle genes, p63 is shown to directly regulate genes involved in glycolytic metabolism in human keratinocytes, such as hexokinase 2 (HK2) and 6-phosphofructo-2-kinase/ fructose-2,6-bisphosphatase 3 (PFKFB3) [51, 52]. HK2 phosphorylates glucose to produce glucose-6-phosphate, representing the first rate-limiting step in glucose metabolism pathways [53]. PFKFB3 is a key regulator that promotes glycolysis. Downregulation of p63 resulted in reduced expression of HK2 and PFKFB3 and concomitant decrease of glycolysis and cell proliferation. These data showed that p63 plays a role in keratinocyte metabolism, a novel mechanism to maintain high proliferation in keratinocytes [54].

The identification of p63 target genes has been a major effort in the field, either through dedicated candidate gene studies or through genome-wide explorative approaches. These studies identify the genomic binding regions of p63 and of p63-regulated genes most often by either knock-down or overexpression of p63 [47, 55], as discussed previously in this review. Some target genes are identified in the context of p63 mutation-associated diseases [56]. It should be noted that p63 binding in human and mouse keratinocytes shares only limited conservation [57]. Epidermal developmentrelated genes seem to be regulated by conserved p63-bound genomic regions, whereas divergent p63-bound regions are involved in metabolic pathways. The distinct regulation controlled by p63 in human and mouse keratinocytes probably reflects the anatomical differences between human and mouse skin.

As a master regulator of epidermal development, p63 regulates transcriptional programs not only through its target genes but also via higher order regulation. In recent years, a number of studies have shown that p63 plays a role in epigenetic regulation in epidermal keratinocytes via several independent mechanisms. p63 can recruit HDAC1/2 to control the repression of cell cycle arrest genes, such as p21 and $14-3-3 \sigma$ [46]. p63 was shown to be a repressor rather than an activator for cell cycle arrest genes, consistent with positive regulation controlled by p63 in keratinocyte proliferation. Furthermore, several chromatin factors are direct p63 target genes [11-13]. Among these factors, SATB1 regulates largescale chromatin remodeling in specific cell types [58, 59]. In keratinocytes, SATB1 binds to the Epidermal Differentiation Complex (EDC) locus and compresses the conformation of EDC where many epidermal terminal differentiation genes are located [11]. In mice, overexpression of Satb1 can partially rescue the phenotype of p63-deficient mouse skin [11]. Another p63 direct target, BRG1, is an ATP-dependent chromatin remodeler. In keratinocytes, BRG1 promotes relocation of the EDC locus from the nuclear periphery towards nuclear interior, which is associated with upregulation of genes in the EDC locus [12]. A chromobox protein, CBX4, that is a component of Polycomb Repressive Complex 1 (PRC1), is also regulated directly by p63 [13]. CBX4 seems to mediate the p63-dependent repression of non-epithelial genes, and overexpression of CBX4 can rescue p63 deficiency in keratinocytes. Chromatin remodelers that are direct p63 targets also include LSH, which regulates DNA methylation and transcriptional silencing [60]. LSH seems to act in p63-mediated senescence. Recently, p63 was shown to regulate the expression of nuclear envelop-associated components (Lamin B1, Lamin A/C, SUN1, Nesprin-3, Plectin) [16]. Several nuclear shape-associated genes, such as Sun1, Syne 3 and Plec, were shown to be p63 direct targets. In the 
epidermis of p63 complete knockout mice, these genes were downregulated, and skin epithelial cells displayed an altered nuclear shape. Consistently, keratinocyte-specific gene loci were relocated away from sites of active transcription toward the heterochromatin-enriched repressive nuclear compartments in $\mathrm{p} 63$ depleted cells.

In addition to regulating chromatin factors and nuclear envelop-associated components involved in higher order gene regulation in keratinocytes, it has become evident that p63 plays a master role in regulating enhancers. It should be noted that many studies discussed below took unbiased genomic approaches rather than p63-centered approaches, yet they came to the conclusion that p63 is the most significant transcription factor that controls epidermal enhancers. Bao et al. showed that genomic regions of p63-bound enhancers detected in keratinocytes are nucleosome-enriched and inaccessible in cells where p63 is not expressed (Fig. 2a), and the cooperation of p63 and BAF is required for opening chromatin (Fig. 2b, c) [61]. This suggests that $\mathrm{p} 63$ and probably its co-regulators have a pioneer factor-like function during epidermal development. Pioneer factors are a class of transcription factors that are the first to engage compact chromatin and to create an accessible chromatin and epigenetic environment for other TFs to be recruited to enhancers [62-64]. They bind to target DNA sites before the onset of transcription, and often pioneer factor-bound regions are open chromatin regions in cells where these factors are expressed and are otherwise closed in cells where they are not expressed. Pioneer factors have been shown to be important for cell identity and commitment. Consistent with this notion, another study on p53 reported that, in addition to binding to the usual active promoters and enhancers of p53-regulated genes, p53 can also bind to inaccessible regions of the chromatin in lung fibroblasts [65]. Interestingly, these inactive regions in lung fibroblasts are seemingly active in two epithelial cells, normal human epithelial keratinocytes (NHEK) and human mammary epithelial cells (HMEC), but are repressed in other cell types. As p63 and p53 share high sequence and structure homology in their DNA-binding domains, and they bind to essentially the same binding motifs on DNA, it has been argued that these 'proto-enhancers' bound by p53 can be active enhancers regulated by p63 or by both p53 and p63 in epithelial cells. Therefore, p53 and p63 may function as pioneer factors. In agreement, epigenomic profiling analyses by ChIP-seq of histone enhancer marks and p63 [14, 29] and by mapping of DNA (hydroxy) methylation sites [15] during keratinocyte differentiation showed that epidermal enhancers are most significantly enriched for p63-binding motifs and that p63 binds mainly to enhancers. p63-bound enhancers are not always active in regulating nearby genes (Fig. 2b). It has been proposed that these p63-bound genomic loci are bookmarked by p63. In particular epithelial cell types or at developmental stages, p63 cooperates with specific transcription factors to activate gene expression in a cell type- and developmental stage-specific manner (Fig. 2c) [14, 50]. Taken together, these observations support an appealing model where p63, probably together with co-regulating transcription and chromatin factors, such as BAF [61], acts as a pioneer factor to open chromatin in epithelial cells and shapes the enhancer landscape.
Fig. 2 The bookmarking role of p63 in epithelial cells. a In the absence of $\mathrm{p} 63$, either in embryonic stem cells before epidermal commitment or in non-epithelial somatic cells, loci of p63-dependent genes remain closed and are occupied by nucleosomes. b, $\mathbf{c}$ In epithelial cells where p63 is expressed, p63 and co-regulators such as BAF can engage closed chromatin and promote its opening. In specific (embryonic) epithelial cell types, some genomic loci are bookmarked by p63 but nearby genes are not activated by $\mathrm{p} 63$. These open chromatin sites are marked by H3K4me1 (b). Other loci and genes are bookmarked and activated by p63. These regions are marked by active enhancer mark $\mathrm{H} 3 \mathrm{~K} 27 \mathrm{ac}(\mathbf{c})$ a Cells without p63 expression
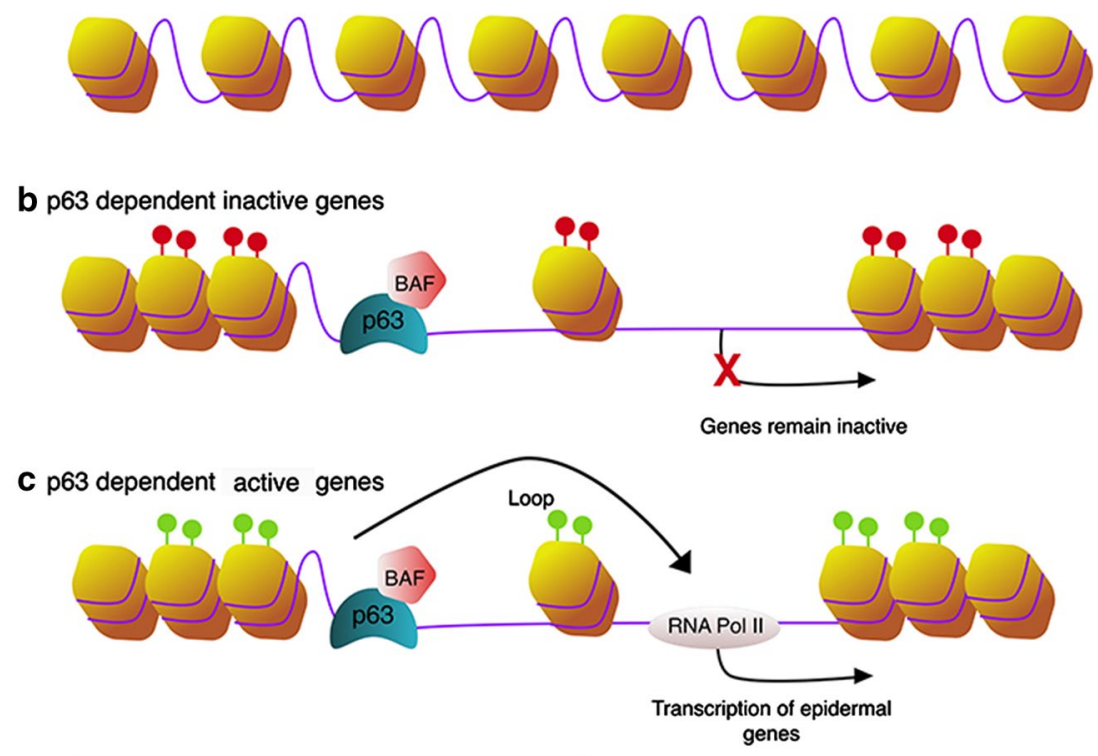

H3K27ac

H3K4me1 
In summary, p63 is likely to regulate gene expression in epidermal keratinocytes at several levels: it regulates direct target genes involved in keratinocyte proliferation and differentiation; it directly regulates chromatin and epigenetic factors; and it might act as a pioneer factor to shape the chromatin and enhancer landscape, and thereby regulate global gene expression.

\section{p63 is essential for proper epidermal commitment}

Consistent with the recent proposed pioneer role of p63 in epidermal development, classical studies in mice nearly two decades ago showed that p63 is essential for proper epidermal commitment $[1,3]$. Two different groups reported that mouse models lacking p63 show striking defects of the epidermis including either partial [1] or complete [3] absence of stratified epithelia. During early vertebrate development, the surface epithelium is composed of a single layer of $\mathrm{K} 8$ / K18-positive ectodermal cells [66]. This single-layered epithelia is functionally important for cellular diffusion, secretion or absorption, but not directly relevant for barrier function [67]. As development proceeds, these ectodermal cells develop into keratinocytes that have high expression of mature epithelial markers, such as K5/K14 and can further develop to the epidermis that has a barrier function. This physiological transition is called epidermal commitment. During this process, proper p63 expression and function is essential for correct commitment by activating epidermal genes and repressing genes of other lineages, and therefore p63 has been proposed to be the 'gatekeeper' of the epithelial lineage [68]. Studies using in vivo and in vitro models and p63-regulated genes are discussed here.

Recently, spatio-temporal expression of p63 during normal mouse embryonic development was analyzed [26]. p63 expression was detected in ectodermal cells near the newly formed somites and the posterior part of the E8.5 embryo, preceding epidermal commitment. Subsequently, p63 expression was enriched at branchial arches and the limb buds, and then expanded to the whole surface of the embryo. The embryonic expression pattern of p63 correlates remarkably well with affected structures, such as the epidermis, orofacial regions and limbs in p63 knockout mice and in p63 mutation-associated human diseases. In normal mice, the single-layered epithelium eventually develops into stratified epithelium, whereas the surface ectoderm of p63 null mouse strains remains a monolayer of non-proliferating cells expressing K8/K18 [68]. Interestingly, genetic complementation with $\Delta \mathrm{Np} 63$ in p63 null mice rescues the development of the skin through activation of epidermal genes, such as the direct p63 target K14 [69], emphasizing the important role of $\Delta \mathrm{Np} 63$ in epidermal development.
Consistently, in vitro studies using mouse embryonic stem cells (mESCs) showed that $\mathrm{p} 63$ expression is detected before expression of the epidermal marker K14 [70-73]. p63 deficiency in mESCs impedes progression towards the stratified epithelial fate, and p63-deficient cells have abnormally upregulated mesodermal genes [6,73], indicating loss of the epithelial cell fate. Furthermore, ectopic expression of $\triangle \mathrm{Np63}$ in mESC-derived p63-deficient ectodermal cells can induce epidermal commitment and lead to differentiation into keratinocytes $[6,70]$. It has become evident that p63 induces expression of many epidermal genes, such as K14, K17 and S100A11, by directly binding to these gene loci [68]. However, it is also important to investigate whether repressed expression of genes from other lineages is directly regulated by $\mathrm{p} 63$ or through p63-related higher order regulation. In summary, these in vivo and in vitro studies support the master regulatory role of p63 in epidermal commitment.

Although the essential role of p63 in epidermal commitment has been established, the gene networks and regulatory mechanisms of epidermal commitment that are upstream and downstream of p63 are not yet fully understood. A closer look into molecular pathways during epidermal development reveals the interaction of p63 and bone morphogenetic protein (BMP) signaling [74]. The $\Delta \mathrm{Np} 63$ isoform activates BMP signaling through direct binding in conserved regulatory regions on the Smad7 promoter, thereby repressing its expression. p63 sustains Bmp7 expression in the epidermis and indirectly controls Bmp4 expression in the dermis. Equally important, during early embryonic development, BMP signaling acts as an epidermal inducer by suppressing the neural fate, probably via p63-regulated gene expression $[66,75,76]$. Furthermore, p63 expression is regulated in a positive autoregulatory manner through a long-range enhancer (p63LRE) [77]. This enhancer is conserved in both human and mouse and is also bound by several other transcription factors including AP2, Cebpa, Cebpb and the POU domain-containing protein Pou3f1. Among them, Cebpa, Cebpb and Pou3f1 repress p63 expression [77]. It has also been reported that Cebpb binds to the $\mathrm{p} 63$ promoter to repress its expression [78]. These studies help unravel the p63-related regulatory network controlling epidermal cell fate. However, continuing efforts to comprehensively and systematically identify p63-dependent transcriptional networks are warranted.

Taken together, in vivo and in vitro studies have demonstrated that p63 is essential for epidermal commitment. The molecular pathways and gene regulatory mechanisms that act during epidermal commitment remain to be elucidated. It will be particularly interesting to investigate whether p63 functions as a pioneer factor during this process, either alone or most probably together with other co-regulators, and how p63 mutations affect this process. 


\section{p63 in cellular reprogramming}

Somatic cell transdifferentiation is a process in which transient ectopic expression of lineage master regulators can induce conversion into a different somatic cell fate without passing through a pluripotent configuration [79-81]. Given the master regulatory role of p63 for proper epidermal commitment and maintenance, hypotheses have arisen that its ectopic expression in non-epithelial cell types might be sufficient to convert these cells to the epidermal fate. This has been demonstrated in a report where the combination of KLF4 with $\Delta$ Np63 could induce the conversion of human fibroblasts to keratinocyte-like cells [82] (Fig. 3a). The transdifferentiated cells showed positive staining for the basal marker K14 and were negative for fibroblast markers, such as MME and COL11A1. In the same study, cancer cells could also be converted to keratinocytes using KLF4 and $\mathrm{p} 63$, suggesting that these two transcription factors are capable of reprogramming cells to the epidermal fate even in different epigenetic backgrounds. Another example is the cooperation between p63 and PAX6 in driving limbal stem cell (LSC) commitment [83] (Fig. 3b). p63 is expressed in both epidermal keratinocytes and LSCs, whereas PAX6 is specific for LSCs. Epidermal keratinocytes and LSCs undergo distinct transcriptional programs during terminal differentiation, with an induction of K1/10 or K3/12 expression for epidermal keratinocytes or LSCs, respectively. Ouyang et al. showed that $\mathrm{p} 63$ was essential for both epidermal keratinocyte and LSC fates, and the combination of p63 and PAX6 defined the LSC fate, consistent with expression patterns of these two genes. Interestingly, LSCs with knock-down of PAX6 acquired the epidermal keratinocyte property of K1/ a

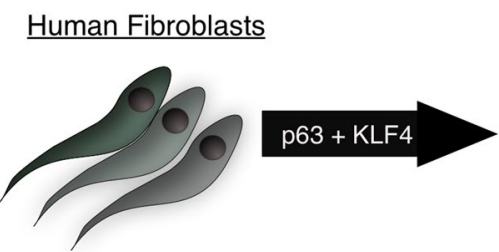

b

Keratinocytes

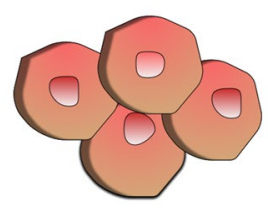

High endogenous p63 expression

\section{PAX6}

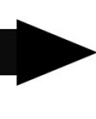

Limbal Stem Cells

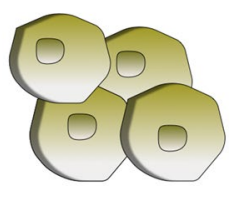

Keratinocytes

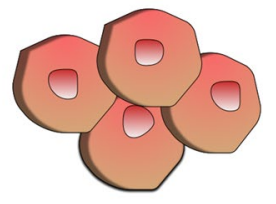

Fig. 3 p63-dependent transdifferentiation. a Human fibroblasts can be reprogrammed into Keratinocyte-like cells through ectopic expression of p63 and KLF4. b Keratinocytes that have high endogenous p63 expression can be reprogrammed into limbal stem cells by ectopic PAX6 expression
K10 induction during terminal differentiation; in contrast, ectopic expression of PAX6 in epidermal keratinocytes gave rise to LSC-like cells. This report is in line with previous studies where reported mutations in either in PAX6 [84] or p63 [85] lead to blindness resulting from limbal stem cell deficiency.

Two studies have also suggested that p63 plays a role in cell reprogramming to a pluripotent cell state. In one study, the authors reported that p63 enables reprogramming to pluripotency in mouse embryonic fibroblasts [86]. In this case, p63 did not induce reprogramming per se, but was important for maximal reprogramming efficiency. On the contrary, another report showed that p63 knockout mouse keratinocytes acquired some pluripotent signatures [87], suggesting that $\mathrm{p} 63$ blocks reprogramming to pluripotency. As p63 is normally not expressed in pluripotent stem cells, the function of p63 in pluripotency is completely unknown. Whether and how $\mathrm{p} 63$ plays a role in cell reprogramming to pluripotency requires further investigation.

So far, gene regulatory programs during these cell fate conversions have not yet been identified. The precise role of p63 in gene regulation during cellular reprogramming, especially towards an epithelial fate or during a switch between different epithelial fates, is important for understanding its master regulatory function controlling epithelial cell identity. Beyond exploring the fundamental biological role of p63, the examples discussed in this review also hold great potential for developing therapeutic options, e.g. converting human epidermal keratinocytes to LSCs for cornea regeneration or to other rare epithelial types.

\section{Potential therapeutic approaches}

Other than cosmetic surgery and correction [88], there is no cure for p63 mutation-associated disorders. Recent improvements in modeling human diseases have opened new perspectives on amelioration of these diseases.

Initially designed to treat cancers, the small compound APR-246/PRIMA-1 ${ }^{\text {MET }}$ was shown to interact with the mutant p53 core domain and promote its folding to the wildtype conformation $[89,90]$. This compound has been tested in a phase I/II clinical trial in patients with either hematological malignancies or hormone-refractory prostate cancer [91]. Given the high degree of homology in the DNA-binding domain between p53 and p63 [92], the potential of APR246 for restoring mutant $\mathrm{p} 63$ conformation and function was tested in two cellular models: epidermal stratification and corneal commitment $[73,93]$. Shen et al. reported that APR246 rescued differentiation defects of keratinocytes established from EEC patients carrying p63 mutations R204W and R304W in the DNA-binding domain, specifically in terms of cell morphology and gene expression in 2D and 
3D epidermal stratification models [93]. A set of epidermal differentiation markers, such as K10, TGM, CysME, LCE2, and Filaggrin, were efficiently induced in APR-246-treated p63 mutant keratinocytes, approaching similar expression levels as observed in wild-type p63 keratinocytes. In addition, p63 target genes, such as ADH7 and Claudin-1, which were downregulated in p63 mutant keratinocytes, were upregulated upon APR-246 treatment. Another report using human-induced pluripotent stem cells (hiPSCs) established from the same EEC patients showed corneal epithelial cells derived from these patient hiPSCs responded to APR-246, and impaired corneal epithelial differentiation was rescued [73]. As p63 EEC mutations likely have a dominant negative effect towards the wild-type protein (Fig. 4a, b), APR-246 can probably restore mutant p63 to the wild-type conformation, thereby rescuing p63 DNA-binding and target gene activation (Fig. 4c). Further in-depth cellular and molecular analyses, such as RNA-seq and ChIP-seq, should be performed to comprehensively evaluate the effect and underlying mechanism of APR-246 on cell differentiation. Ultimately, in vivo studies and clinical trials are necessary to explore the therapeutic potential of APR-246.

In addition to small compounds, small interfering RNAs (siRNA) have also been explored for their therapeutic potential in treating p63 mutation-associated diseases. Novelli et al. reported the identification of a panel of siRNAs specifically targeting the p63 EEC R304W mutant transcripts. These R304W-targeting siRNAs can rescue defects during corneal epithelial differentiation of hiPSCs carrying the R304W mutation [94]. Similarly, the siRNA approach has also been applied to specifically target the p63 EEC R279H transcripts in oral mucosa epithelial stem cells (OMESCs) from EEC patients. Treatment with these siRNAs restored the epithelial differentiation capacity of these patient cells [95]. The success of these siRNA approaches also confirms the dominant negative model of p63 EEC mutations and demonstrates that degrading the mutant allele alone is sufficient to rescue the epithelial defects caused by these mutations (Fig. 4d).

In addition to approaches discussed in this review, rapidly emerging technological tools, such as genome editing, have provided new possibilities for developing therapeutic options for p63 mutation-associated diseases. Further in vivo studies and clinical trials are necessary to validate these therapeutic options and eventually to treat patients.

\section{Conclusions and future perspectives}

Since the discovery of the transcription factor p63 nearly two decades ago, its master regulatory role in the development of the epidermis has been extensively studied in cellular and animal models and at normal and diseased states. Although many isoforms have been identified for the p63 protein, the $\Delta \mathrm{Np} 63 \alpha$ is the most abundant isoform in epidermal cells and is probably responsible for the bulk of the functional p63 activity. p63 plays its key role in epidermal commitment and terminal differentiation, not only by regulating its direct target genes, but also by shaping the chromatin state, in particular, the enhancer landscape. Recent in-depth molecular analyses suggest that $\mathrm{p} 63$ functions as one of the pioneer factors for epidermal cell identity. This model is supported by
Fig. 4 Potential therapeutic approaches targeting p63 mutations. a In normal epidermal cells, p63 can bind to promoter and enhancer regions in the genome and regulate expression of its target genes. b Mutations in the DNA-binding domain of p63 (EEC syndrome mutations) disrupt p63 DNA-binding, thereby deregulating its target genes. c Silencing the mutant allele of EEC syndrome mutations by mutation-specific siRNAs allows wild-type p63 binding and activation of p63 target genes. $\mathbf{d}$ The use of the small molecular compound APR-246/ PRIMA-1 ${ }^{\text {MET1 }}$ possibly changes the conformation of mutant p63 protein and restores its ability to bind and activate its target genes a

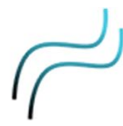

b

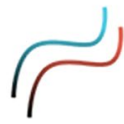

C

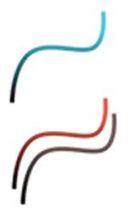

d

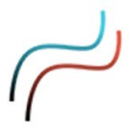

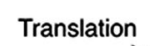
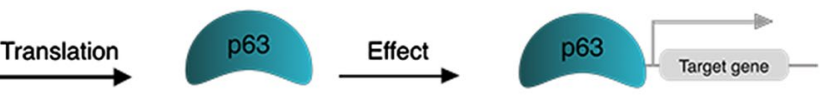

p63
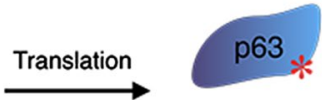

Altered DNA binding domain
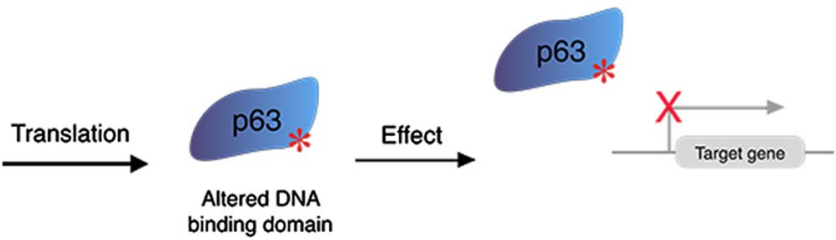

Translation
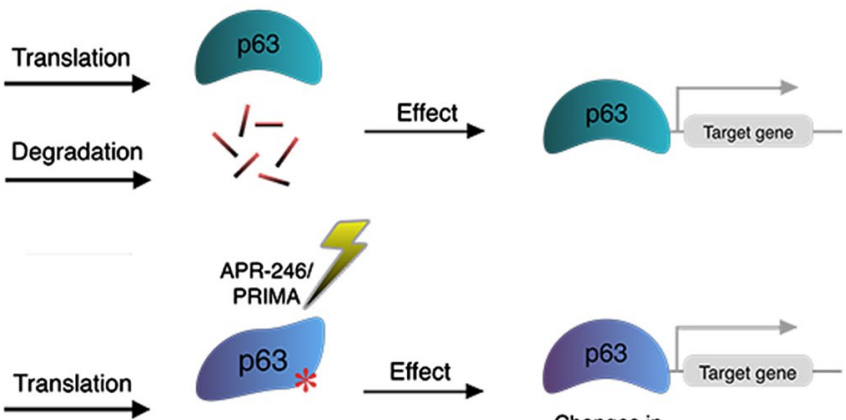
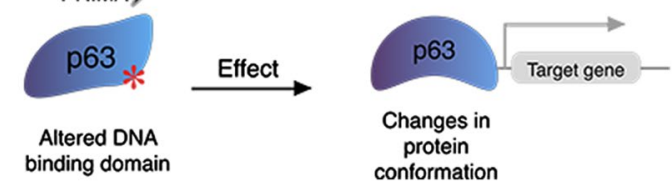
classical cellular and in vivo studies as well as recent transdifferentiation studies where p63 is required for epithelial cell lineages. Testing this pioneer factor model during epidermal commitment at the molecular level and identifying key regulators that cooperate with p63 are critical topics for future research. It is plausible that p63 mutations involved in human disorders may deregulate gene expression by disrupting the pioneer factor function and thereby the epithelial epigenetic landscape, subsequently giving rise to epidermal phenotypes. Current and ongoing efforts to understand the role of p63 in normal and diseased conditions are not only important for the fundamental insights into gene regulation and epidermal development, but also for the development of novel therapeutic strategies to treat patients with p63 mutation-associated and other related diseases. Although therapeutic development for these diseases is still at its infancy, encouraging results of small molecule compounds and siRNA approaches have opened new possibilities, and should be extended further to eventually benefit patients.

Acknowledgements This research was supported by NWO/ALW/ MEERVOUD/836.12.010 (H. Z.), Radboud University fellowship (H. Z.), Brazilian Science without Borders program (E. S.). We thank Matthew Makowski for critical review of this manuscript and Hans van Bokhoven for discussion.

Open Access This article is distributed under the terms of the Creative Commons Attribution 4.0 International License (http://creativecommons.org/licenses/by/4.0/), which permits unrestricted use, distribution, and reproduction in any medium, provided you give appropriate credit to the original author(s) and the source, provide a link to the Creative Commons license, and indicate if changes were made.

\section{References}

1. Mills AA, Zheng B, Wang XJ et al (1999) p63 is a p53 homologue required for limb and epidermal morphogenesis. Nature 398:708-713

2. Yang A, Kaghad M, Wang Y et al (1998) p63, a p53 homolog at $3 q 27-29$, encodes multiple products with transactivating, deathinducing, and dominant-negative activities. Mol Cell 2:305-316

3. Yang A, Schweitzer R, Sun D et al (1999) p63 is essential for regenerative proliferation in limb, craniofacial and epithelial development. Nature 398:714-718

4. Duijf PHG, Van Bokhoven H, Brunner HG (2003) Pathogenesis of split-hand/split-foot malformation. Hum Mol Genet 12:51-60

5. Rinne T, Brunner HG, Van Bokhoven H (2007) P63-associated disorders. Cell Cycle 6:262-268

6. Medawar A, Virolle T, Rostagno P et al (2008) $\Delta$ Np63 is essential for epidermal commitment of embryonic stem cells. PLoS One 3:e3441

7. Thomason HA, Zhou H, Kouwenhoven EN et al (2010) Cooperation between the transcription factors p63 and IRF6 is essential to prevent cleft palate in mice. J Clin Invest 120:1561-1569

8. Carroll DK, Carroll JS, Leong C-O et al (2006) P63 regulates an adhesion programme and cell survival in epithelial cells. Nat Cell Biol 8:551-561
9. Pellegrini G, Dellambra E, Golisano O et al (2001) p63 identifies keratinocyte stem cells. Proc Natl Acad Sci USA 98:3156-3161

10. Truong AB, Kretz M, Ridky TW et al (2006) p63 regulates proliferation and differentiation of developmentally mature keratinocytes. Genes Dev 20:3185-3197

11. Fessing MY, Mardaryev AN, Gdula MR et al (2011) P63 regulates Satb1 to control tissue-specific chromatin remodeling during development of the epidermis. J Cell Biol 194:825-839

12. Mardaryev AN, Gdula MR, Yarker JL et al (2014) p63 and Brg1 control developmentally regulated higher-order chromatin remodelling at the epidermal differentiation complex locus in epidermal progenitor cells. Development 141:3437-3437

13. Mardaryev AN, Liu B, Rapisarda V et al (2016) Cbx4 maintains the epithelial lineage identity and cell proliferation in the developing stratified epithelium. J Cell Biol 212:77-89

14. Kouwenhoven EN, Oti M, Niehues H et al (2015) Transcription factor p63 bookmarks and regulates dynamic enhancers during epidermal differentiation. EMBO Rep 16:863-878

15. Rinaldi L, Datta D, Serrat J et al (2016) Dnmt3a and Dnmt3b associate with enhancers to regulate human epidermal stem cell homeostasis. Cell Stem Cell 19:491-501

16. Rapisarda V, Malashchuk I, Asamaowei IE et al (2017) p63 transcription factor regulates nuclear shape and expression of nuclear envelope-associated genes in epidermal keratinocytes. J Invest Dermatol 137:2157-2167

17. Schmale H, Bamberger C (1997) A novel protein with strong homology to the tumor suppressor p53. Oncogene 53:1363-1367

18. Serber Z, Lai HC, Yang A et al (2002) A C-terminal inhibitory domain controls the activity of p63 by an intramolecular mechanism. Am Soc Microbiol 22:8601-8611

19. Rinne T, Clements SE, Lamme E et al (2008) A novel translation re-initiation mechanism for the p63 gene revealed by aminoterminal truncating mutations in Rapp-Hodgkin/Hay-Wells-like syndromes. Hum Mol Genet 17:1968-1977

20. Mangiulli M, Valletti A, Caratozzolo MF et al (2009) Identification and functional characterization of two new transcriptional variants of the human p63 gene. Nucleic Acids Res 37:6092-6104

21. Rizzo JM, Romano R-A, Bard J, Sinha S (2015) RNA-seq studies reveal new insights into p63 and the transcriptomic landscape of the mouse skin. J Invest Dermatol 135:629-632

22. Sethi I, Romano R-A, Gluck C et al (2015) A global analysis of the complex landscape of isoforms and regulatory networks of p63 in human cells and tissues. BMC Genomics 16:584

23. Koster MI, Kim S, Mills AA et al (2004) p63 is the molecular switch for initiation of an epithelial stratification program. Genes Dev 18:126-131

24. King KE, Ponnamperuma RM, Gerdes MJ et al (2006) Unique domain functions of p63 isotypes that differentially regulate distinct aspects of epidermal homeostasis. Carcinogenesis 27:53-63

25. Pellegrini G, Rama P, Matuska S et al (2013) Biological parameters determining the clinical outcome of autologous cultures of limbal stem cells. Regen Med 8:553-567

26. Zhao Q, Liu S, Zhang H et al (2015) Spatiotemporal expression of p63 in mouse epidermal commitment. Int J Mol Sci $16: 29542-29553$

27. Romano R-A, Smalley K, Magraw C et al (2012) Np63 knockout mice reveal its indispensable role as a master regulator of epithelial development and differentiation. Development 139:772-782

28. Deutsch GB, Zielonka EM, Coutandin D et al (2011) DNA damage in oocytes induces a switch of the quality control factor TAp63 $\alpha$ from dimer to tetramer. Cell 144:566-576

29. Cavazza A, Miccio A, Romano O et al (2016) Dynamic transcriptional and epigenetic regulation of human epidermal keratinocyte differentiation. Stem Cell Rep 6:618-632 
30. Terrinoni A, Serra V, Bruno E et al (2013) Role of p63 and the Notch pathway in cochlea development and sensorineural deafness. Proc Natl Acad Sci USA 110:7300-7305

31. Cefalù S, Lena AM, Vojtesek B et al (2015) Tap63gamma is required for the late stages of myogenesis. Cell Cycle 14:894-901

32. Rouleau M, Medawar A, Hamon L et al (2011) TAp63 is important for cardiac differentiation of embryonic stem cells and heart development. Stem Cells 29:1672-1683

33. Su X, Paris M, Gi YJ et al (2009) TAp63 prevents premature aging by promoting adult stem cell maintenance. Cell Stem Cell 5:64-75

34. Su X, Gi YJ, Chakravarti D et al (2012) TAp63 is a master transcriptional regulator of lipid and glucose metabolism. Cell Metab 16:511-525

35. Suh E, Yang A, Kettenbach A et al (2006) p63 protects the female germ line during meiotic arrest. Nature 444:624-628

36. Celli J, Duijf P, Hamel BCJ et al (1999) Heterozygous germline mutations in the p53 homolog p63 are the cause of EEC syndrome. Cell 99:143-153

37. Rökaeus N, Shen J, Eckhardt I et al (2010) PRIMA-1(MET)/APR246 targets mutant forms of p53 family members p63 and p73. Oncogene 29:6442-6451

38. Browne G, Cipollone R, Lena AM et al (2011) Differential altered stability and transcriptional activity of $\Delta \mathrm{Np} 63$ mutants in distinct ectodermal dysplasias. J Cell Sci 124:2200-2207

39. Rinne T, Spadoni E, Kjaer KW et al (2006) Delineation of the ADULT syndrome phenotype due to arginine 298 mutations of the p63 gene. Eur J Hum Genet 14:904-910

40. Evans JA (2007) Diaphragmatic defects and limb deficienciestaking sides. Am J Med Genet 140A:1396-1406

41. Chitayat D, Babul R, Silver MM et al (1996) Terminal deletion of the long arm of chromosome 3 [46, XX, de1 (3)(427 - > qter)]. Am J Med Genet 61:45-48

42. Vernersson-Lindahl E, Garcia EL, Mills AA (2013) An allelic series of Trp63 mutations defines TAp63 as a modifier of EEC syndrome. Am J Med Genet 164A:1961-1971

43. Ferone G, Thomason HA, Antonini D et al (2012) Mutant p63 causes defective expansion of ectodermal progenitor cells and impaired FGF signalling in AEC syndrome. EMBO Mol Med 4:192-205

44. Truong AB, Khavari PA (2007) Control of keratinocyte proliferation and differentiation by p63. Cell Cycle 6:295-299

45. Westfall MD, Mays DJ, Sniezek JC, Pietenpol JA (2003) The Np63 phosphoprotein binds the $\mathrm{p} 21$ and 14-3-3 promoters in vivo and has transcriptional repressor activity that is reduced by HayWells syndrome-derived mutations. Mol Cell Biol 23:2264-2276

46. LeBoeuf M, Terrell A, Trivedi S et al (2010) Hdac1 and Hdac2 act redundantly to control p63 and p53 functions in epidermal progenitor cells. Dev Cell 19:807-818

47. Della Gatta G, Bansal M, Ambesi-Impiombato A et al (2008) Direct targets of the TRP63 transcription factor revealed by a combination of gene expression profiling and reverse engineering. Genome Res 18:939-948

48. Ihrie RA, Marques MR, Nguyen BT et al (2005) Perp is a p63-regulated gene essential for epithelial integrity. Cell 120:843-856

49. Romano R-A, Birkaya B, Sinha S (2007) A functional enhancer of Keratin14 is a direct transcriptional target of $\Delta \mathrm{Np} 63$. J Invest Dermatol 127:1175-1186

50. Kouwenhoven EN, van Bokhoven H, Zhou H (2015) Gene regulatory mechanisms orchestrated by p63 in epithelial development and related disorders. Biochim Biophys Acta Gene Regul Mech 1849:590-600

51. Viticchiè G, Agostini M, Maria A et al (2015) p63 supports aerobic respiration through hexokinase II. Proc Natl Acad Sci USA 112:1-6

52. Hamanaka RB, Mutlu G (2017) PFKFB3, a direct target of p63, is required for proliferation and inhibits differentiation in epidermal keratinocytes. J Invest Dermatol 137:1267-1276
53. Ahn KJ, Kim J, Yun M et al (2009) Enzymatic properties of the $\mathrm{N}$ - and C-terminal halves of human hexokinase II. BMB Rep $14: 350-355$

54. Amelio I, Melino G, Candi E (2017) p63 adjusts sugar taste of epidermal layers. J Invest Dermatol 137:1204-1206

55. Yang A, Zhu Z, Kapranov P et al (2006) Relationships between p63 binding, DNA sequence, transcription activity, and biological function in human cells. Mol Cell 24:593-602

56. Sen GL, Boxer LD, Webster DE et al (2012) ZNF750 is a p63 target gene that induces KLF4 to drive terminal epidermal differentiation. Dev Cell 22:669-677

57. Sethi I, Gluck C, Zhou H et al (2017) Evolutionary re-wiring of p63 and the epigenomic regulatory landscape in keratinocytes and its potential implications on species-specific gene expression and phenotypes. Nucleic Acids Res 45:8208-8224

58. Nakayama Y, Mian IS, Kohwi-Shigematsu T, Ogawa T (2005) A nuclear targeting determinant for SATB1, a genome organizer in the T cell lineage. Cell Cycle 4:1099-1106

59. Galande S, Purbey PK, Notani D, Kumar PP (2007) The third dimension of gene regulation: organization of dynamic chromatin loopscape by SATB1. Curr Opin Genet Dev 17:408-414

60. Keyes WM, Pecoraro M, Aranda V et al (2011) $\Delta$ Np63 $\alpha$ is an oncogene that targets chromatin remodeler Lsh to drive skin stem cell proliferation and tumorigenesis. Cell Stem Cell 8:164-176

61. Bao X, Rubin AJ, Qu K et al (2015) A novel ATAC-seq approach reveals lineage-specific reinforcement of the open chromatin landscape via cooperation between BAF and p63. Genome Biol 16:284

62. Soufi A, Garcia MF, Pellegrini M et al (2015) Pioneer transcription factors target partial DNA motifs on nucleosomes to initiate reprogramming pioneer transcription factors target partial DNA motifs on nucleosomes to initiate reprogramming. Cell 161:555-568

63. Zaret KS, Carroll JS (2011) Pioneer transcription factors: establishing competence for gene expression. Genes Dev 25:2227-2241

64. Hsu H-T, Yang Z, Wang J et al (2015) Recruitment of RNA polymerase II by the pioneer transcription factor PHA-4. Science 348:1372-1376

65. Sammons MA, Zhu J, Drake AM, Berger SL (2015) TP53 engagement with the genome occurs in distinct local chromatin environments via pioneer factor activity. Genome Res 25:179-188

66. Aberdam D (2008) Epidermal stem cell fate: what can we learn from embryonic stem cells? Cell Tissue Res 331:103-107

67. Koster MI, Roop DR (2004) Genetic pathways required for epidermal morphogenesis. Eur J Cell Biol 83:625-629

68. Shalom-Feuerstein R, Lena AM, Zhou H et al (2011) $\Delta$ Np63 is an ectodermal gatekeeper of epidermal morphogenesis. Cell Death Differ 18:887-896

69. Candi E, Rufini A, Terrinoni A et al (2006) Differential roles of p63 isoforms in epidermal development: selective genetic complementation in p63 null mice. Cell Death Differ 13:1037-1047

70. Aberdam D, Gambaro K, Rostagno P et al (2007) Key role of p63 in BMP-4-induced epidermal commitment of embryonic stem cells. Cell Cycle 6:291-294

71. Tadeu AMB, Horsley V (2013) Notch signaling represses p63 expression in the developing surface ectoderm. Development 140:3777-3786

72. Bilousova G, Chen J, Roop DR (2011) Differentiation of mouse induced pluripotent stem cells into a multipotent keratinocyte lineage. J Invest Dermatol 131:857-864

73. Shalom-Feuerstein R, Serror L, Aberdam E et al (2013) Impaired epithelial differentiation of induced pluripotent stem cells from ectodermal dysplasia-related patients is rescued by the small compound APR-246/PRIMA-1MET. Proc Natl Acad Sci USA 110:2152-2156

74. De Rosa L, Antonini D, Ferone G et al (2009) P63 suppresses non-epidermal lineage markers in a bone morphogenetic 
protein-dependent manner via repression of Smad7. J Biol Chem 284:30574-30582

75. Hemmati-Brivanlou A, Melton D (1997) Vertebrate neural induction. Annu Rev Neurosci 20:43-60

76. Stern CD (2006) Neural induction: 10 years on since the "default model". Curr Opin Cell Biol 18:692-697

77. Antonini D, Sirico A, Aberdam E et al (2015) A composite enhancer regulates p63 gene expression in epidermal morphogenesis and in keratinocyte differentiation by multiple mechanisms. Nucleic Acids Res 43:862-874

78. Borrelli S, Testoni B, Callari M et al (2007) Reciprocal regulation of p63 by C/EBP delta in human keratinocytes. BMC Mol Biol 28:85

79. Blau HM (1989) How fixed is the differentiated state? Lessons from heterokaryons. Trends Genet 5:268-272

80. Davis RL, Weintraub H, Lassar AB (1987) Expression of a single transfected cDNA converts fibroblasts to myoblasts. Cell 51:987-1000

81. Graf T, Enver T (2009) Forcing cells to change lineages. Nature 462:587-594

82. Chen Y, Mistry DS, Sen GL (2014) Highly rapid and efficient conversion of human fibroblasts to keratinocyte-like cells. J Invest Dermatol 134:335-344

83. Ouyang H, Xue Y, Lin Y et al (2014) WNT7A and PAX6 define corneal epithelium homeostasis and pathogenesis. Nature 511:358-361

84. Secker GA, Daniels JT (2008) Corneal epithelial stem cells: deficiency and regulation. Stem Cell Rev 4:159-168

85. Di Iorio E, Kaye SB, Ponzin D, Barbaro V (2012) Limbal stem cell deficiency and ocular phenotype in ectrodactyly-ectodermal dysplasia-clefting syndrome caused by $\mathrm{p} 63$. Ophthalmology 119:74-83

86. Alexandrova EM, Petrenko O, Nemajerova A et al (2013) D Np63 regulates select routes of reprogramming via multiple mechanisms. Cell Death Differ 20:1698-1708
87. Chakravarti D, Su X, Cho MS et al (2014) Induced multipotency in adult keratinocytes through down-regulation of $\triangle N p 63$ or DGCR8. Proc Natl Acad Sci USA 111:E572-E581

88. Cabiling D, Yan A, McDonald-McGinn DM et al (2005) Cleft lip and palate repair in Hay-Wells/ankyloblepharon-ectodermal dysplasia-clefting syndrome. Cleft Palate Craniofac J 44:335-339

89. Lambert JMR, Moshfegh A, Hainaut P et al (2009) Mutant p53 reactivation by PRIMA-1 MET induces multiple signaling pathways converging on apoptosis. Oncogene 29:1329-1338

90. Lambert JMR, Gorzov P, Veprintsev DB et al (2009) PRIMA-1 reactivates mutant $\mathrm{p} 53$ by covalent binding to the core domain. Cancer Cell 15:376-388

91. Lehmann S, Bykov VJN, Ali D et al (2012) Targeting p53 in vivo: a first-in-human study with p53-targeting compound APR-246 in refractory hematologic malignancies and prostate cancer. J Clin Oncol 30:3633-3639

92. Klein C, Georges G, Künkele KP et al (2001) High thermostability and lack of cooperative DNA binding distinguish the p63 core domain from the homologous tumor suppressor p53. J Biol Chem 276:37390-37401

93. Shen J, van den Bogaard EH, Kouwenhoven EN et al (2013) APR-246/PRIMA-1MET rescues epidermal differentiation in skin keratinocytes derived from EEC syndrome patients with p63 mutations. Proc Natl Acad Sci USA 110:2157-2162

94. Novelli F, Lena AM, Panatta E et al (2016) Allele-specific silencing of EEC p63 mutant R304W restores p63 transcriptional activity. Cell Death Dis 7:e2227

95. Barbaro V, Nasti AA, Raffa P et al (2016) Personalized stem cell therapy to correct corneal defects due to a unique homozygousheterozygous mosaicism of ectrodactyly-ectodermal dysplasiaclefting syndrome. Stem Cells Transl Med 5:1-8 